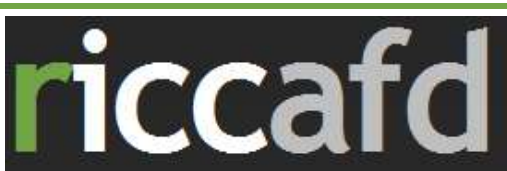

Revista Iberoamericana de Ciencias de la Actividad Física y el Deporte

\title{
TENDENCIAS ACTUALES DE LA ACELEROMETRÍA PARA LA CUANTIFICACIÓN DE LA ACTIVIDAD FÍSICA
}

\section{CURRENT TRENDS OF ACCELEROMETRY TO ASSESS PHYSICAL ACTIVITY}

\section{Santos-Lozano, A. ${ }^{1}$ y Garatachea, N. ${ }^{1}$}

1 Facultad de Ciencias de la Salud y del Deporte. Universidad de Zaragoza. alexsanloz@hotmail.com

2 Facultad de Ciencias de la Salud y Deporte. Universidad de Zaragoza. nuria.garatachea@unizar.es

Código UNESCO: 5802.99

Clasificación Consejo de Europa: 17. Actividad Física y Salud.

Recibido el 15 de junio de 2012

Aceptado el 24 de agosto de 2012

PALABRAS
CLAVE:
Actividad
Física,
Acelerómetro,
Acelerometría,
Monitores de
Actividad

KEY WORDS: Physical Activity, Accelerometers, Accelerometry, Activity Monitors.

\begin{abstract}
RESUMEN
Evaluar la actividad física de forma precisa y fiable sigue siendo un importante reto para los epidemiólogos, científicos, médicos, especialistas en ejercicio e investigadores del comportamiento. Para ello, actualmente, los acelerómetros son dispositivos ampliamente utilizados en investigación científica. En el presente artículo se pretende dar muestra de cuál es la situación actual del uso de la acelerometría para cuantificar niveles de actividad física. Así como sus limitaciones y perspectivas de futuro con el fin de contribuir a su mejor uso.
\end{abstract}

their limitations and future directions in order to promote a better use of this technology. 


\section{INTRODUCCIÓN}

El deseo de lograr comprender mejor la relación entre actividad física y salud, así como poder explicar el drástico aumento en la prevalencia de sobrepeso y obesidad en jóvenes y adultos, ha centrado la atención en la necesidad de mejorar las herramientas utilizadas para cuantificar los niveles de actividad física.

El desarrollo tecnológico ha permitido generar instrumentos fáciles de utilizar y que, de una manera objetiva, valoran el nivel de actividad física. Por ejemplo, en la década de los noventa del pasado siglo XX, el monitor de frecuencia cardíaca fue ampliamente utilizado, siendo el método de elección preferido por muchos investigadores (1) para medir la intensidad de la actividad física por medio de los latidos por minuto del corazón. Sin embargo, se desarrolló el empleo de los podómetros, que miden la actividad física de forma también objetiva contabilizando el número de pasos por día, pero carecen de la posibilidad de cuantificar su intensidad. Por eso, en los últimos años se ha incrementado la popularidad y el empleo de los acelerómetros como herramientas objetivas de cuantificación de la actividad física en distintas poblaciones $(2,3,4)$, proporcionando información relativa sobre la intensidad, la frecuencia y la duración de la actividad física desarrollada de la persona que lleva el monitor.

En este sentido, se pueden realizar algunas reflexiones relativas a los acelerómetros para contextualizar la fase investigadora en la que se encuentran, las futuras direcciones que pueden tomar, conocer sus limitaciones y contribuir a su mejor uso.

\section{ESTADO ACTUAL DE LA INVESTIGACIÓN E INVESTIGADORES MÁS RELEVANTES}

En la década de los años 90, la acelerometría estaba todavía considerada en fase de desarrollo. En 1999 se llevó a cabo en el prestigioso Instituto Cooper el congreso "Measurement of Physical Activity", donde se facilitaron a la comunidad científica algunas conclusiones y recomendaciones futuras sobre el empleo de los acelerómetros. Desde entonces, los investigadores han dedicado grandes esfuerzos para responder a la necesidad de mejorar este campo con los avances tecnológicos y las nuevas aplicaciones de tecnologías existentes y emergentes. La literatura científica publicada proporciona una demostración de su importancia a partir de esta fecha. En la figura 1 se puede observar la tendencia ascendente en el número de publicaciones de artículos científicos que tratan sobre acelerometría desde entonces. Los datos fueron obtenidos mediante búsquedas en la base de datos bibliográfica Medline entre los artículos originales de investigación - revisiones que incluían los términos "acelerometer", "activity monitor", "physical activity", "sport" y/o "exercise" en el título, palabras clave o resumen.

Rev. Ib. CL. Act.Fis. Dep. 


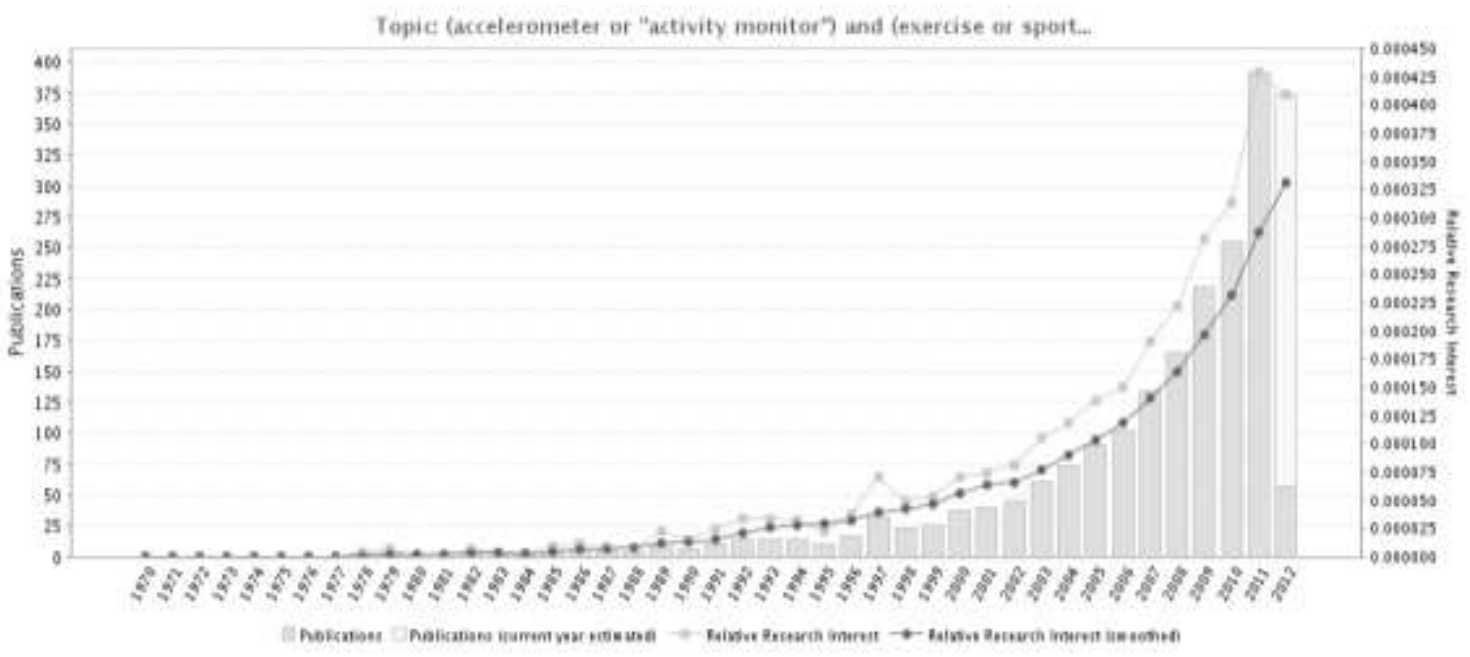

Figura 1. Evolución de las publicaciones a lo largo del tiempo

En la figura 2 se muestran el número de publicaciones relacionadas con acelerometría en revistas internacionales y con factor de impacto, siendo "Medicine and Science in Sport and Exercise" la revista en las que más se han publicado.

Med Sci Sport Exer
J Phys Act Health
Med Sci Sports Exerc
Conf Proc leee Eng Med Biol Soc
Int J Behav Nutr Phys Act
Prev Med
Arch Phys Med Rehabil
Res Q Exercise Sport
Gait Posture
Eur J Appl Physiol
Pediatr Exerc Sci
Bmc Public Health
J Appl Physiol
Am J Prev Med
Pace
Obesity (silver Spring)
Br J Sports Med
J Sci Med Sport
J Biomech
J Rehabil Res Dev

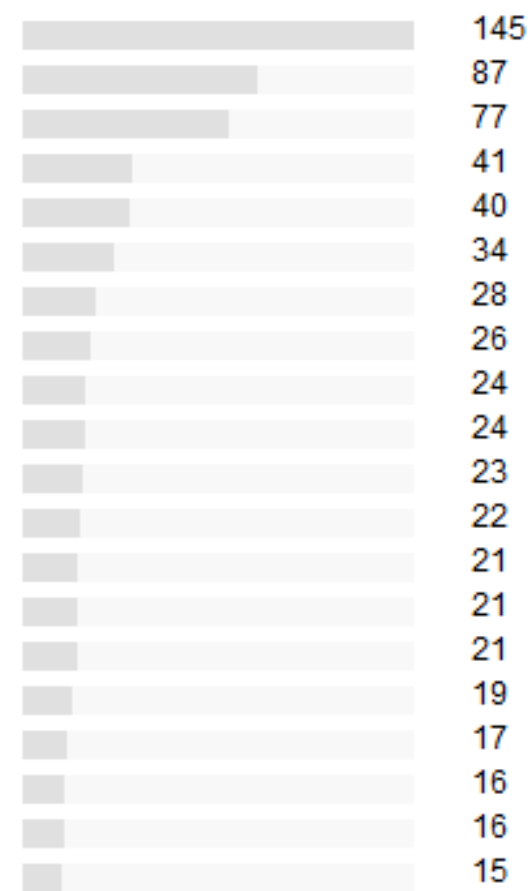

Figura 2. Ranking de revistas que han publicado sobre acelerometría

Por último, en la figura 3 se puede observar los autores que más han publicado en revistas de prestigioso nivel sobre esta temática.

Rev. Ib. CC. Act.Fis. Dep. 


\begin{tabular}{l|r} 
Westerterp K & 40 \\
Sallis J & 23 \\
Pate R & 19 \\
Ainsworth B & 17 \\
Stam H & 16 \\
Ekelund U & 15 \\
Trost S & 14 \\
Freedson P & 14 \\
Andersen L & 13 \\
Guralnik J & 13 \\
Tudor-Locke C & 12 \\
McDermott M & 12 \\
Bassett D & 11 \\
Matthews C & 11 \\
Snook E & 11 \\
Criqui M & 11 \\
Treuth M & 11 \\
McAuley E & 10 \\
Gardner A & 10 \\
Baranowski T & 10
\end{tabular}

Figura 3. Lista de los autores que más han publicado en acelerometría

\section{LIMITACIONES DE LOS - descargar el registro de cada ACTUALES MONITORES \\ dispositivo en cuestión.}

A pesar de las conocidas ventajas de los acelerómetros para medir los niveles de actividad física y estimar gasto energético, es necesario que los investigadores conozcan sus limitaciones.

- Las unidades que los acelerómetros proporcionan como indicador de movimiento (counts) no son equivalentes entre marcas $y$, muchas veces, tampoco entre monitores de la misma. Además, los algoritmos patentados para estimar el gasto calórico (5) son únicos para cada monitor. Esto reduce en muchas ocasiones la posibilidad de comparar entre distintos modelos y marcas existentes en el mercado.

- Cada marca de acelerómetro posee su propio software, que se necesita para iniciar, configurar y
- El tiempo invertido por el personal investigador en procesar y analizar los datos es alto, siendo mayor en sensores múltiples y estudios epidemiológicos (6).

- Se debe entrenar a los usuarios para utilizar el dispositivo de monitorización prestando cuidadosa atención a su colocación (7).

- Es necesario definir el protocolo y la configuración del acelerómetro teniendo en cuenta el tiempo de almacenamiento de la información (epoch), horas de registro diario, número de días y días de la semana (entre semana o fin de semana) en los que se va a analizar el nivel de actividad física (6).

Rev. Ib. CE. Act.Fis. Dep. 
- Una de las principales limitaciones estriba en que la mayoría de los acelerómetros piezoeléctricos son sólo fiables para detectar eventos dinámicos, pudiendo ocurrir un fenómeno denominado leakage. Este se produce cuando el cambio inicial en la carga en el elemento piezoeléctrico del monitor se disipa en el tiempo, incluso si la carga estática que causó el cambio inicial está todavía presente (misma velocidad, aceleración diferente) (8). La frecuencia con la cual se produce este fenómeno va a depender de la constante tiempo, una propiedad física del material piezoeléctrico.

- Así, los acelerómetros piezoeléctricos presentan una incapacidad para detectar el componente estático de la aceleración, esto significa que no son capaces de medir los ángulos con respecto a la gravedad de la superficie y la postura (de pie versus sentado). Sin embargo, un novedoso monitor denominado Activpal, que se coloca en el muslo, tiene un inclinómetro que detecta la postura de la persona (de pie estático, sentado y/o descansando 0 en comportamiento no sedentario). Además, otros monitores consistentes en múltiples sensores proporcionan una definición más precisa de la posición, pero el análisis de los datos es más complicado que el uso de una sola unidad.

- La exactitud y precisión de cada dispositivo depende de una buena calibración de la unidad, lo que reduce la variabilidad intermonitores. Sin embargo, esta unidad de calibración no es la misma con el paso del tiempo, con lo que se necesita calibración periódica. Hay que tener en cuenta que existe una tecnología actual, por medio de un chip integrado, donde la calibración es menos necesaria. Otros aspectos como las caídas, altas y bajas temperaturas y un uso prolongado en el tiempo son factores que disminuyen la calibración correcta del acelerómetro.

Una de las limitaciones más destacadas de los acelerómetros es la imposibilidad de captar algunos tipos de actividad física diaria, fundamentalmente relacionada con los movimientos de las extremidades superiores del cuerpo. Por ejemplo, no es posible detectar movimientos de los brazos mientras se permanece sentado con monitores simples, así como saber si el sujeto monta en bicicleta o levanta de pesas.

A su vez, tanto los sensores simples como múltiples, son ineficaces en la estimación del consumo de energía en determinadas situaciones, como por ejemplo caminar o correr en pendiente o llevar una carga.

El elevado precio de los monitores $(<150 €)$ puede considerarse una limitación. 


\section{Futuras direcciones}

Actualmente existe una tecnología emergente, fruto de la necesidad de mejora. En este sentido, son varios los laboratorios de investigación y empresas que han puesto diversas estrategias en marcha: a) Utilizar monitores con múltiples sensores que se colocan en diferentes partes del cuerpo (tobillos, cintura, muñecas...) y, b) Combinar el acelerómetro con otro sensor fisiológico (frecuencia cardiaca, temperatura...) integrado en un único dispositivo.

Chen y Basset (8), en una interesante revisión, concluyeron que los futuros monitores de acelerometría deberían estar diseñados con el fin de mejorar significativamente su capacidad de predecir el gasto energético. Además, con el fin de ser lo suficientemente portátiles y fuertes para su aplicación en la vida diaria deben seguir dos criterios fundamentales: 1) Deben ser compactos y no tener ningún, o la menor cantidad posible de cables y, 2) Deben poseer una capacidad suficiente de procesamiento de datos y de almacenamiento durante un periodo extenso de tiempo.

\section{Acelerómetros con múltiples sensores}

Esta clase de acelerómetros tienen la capacidad de detectar cambios posturales y movimientos de baja intensidad, principal limitación de los acelerómetros que se portan en la cintura. La aplicación de este tipo de dispositivos se ha realizado principalmente en laboratorios de investigación y están orientados a la rehabilitación de pacientes con amputación de una pierna, cirugía de espalda o insuficiencia cardiaca crónica entre otros trastornos físicos.

Recientemente, ha sido lanzado al mercado un dispositivo inteligente para la estimación de gasto de energía y actividad llamado IDEEA, (IDEEA, MiniSun LLC, Fresno, CA), un nuevo microprocesador portátil de actividad física. EI IDEEA fue diseñado con el fin registrar los movimientos complejos de la actividad física diaria empleando sensores piezoeléctricos en el pecho, muslos y pies. Utiliza sensores de movimiento de pequeño tamaño, con un peso menor de un gramo, que se colocan con cinta hipoalérgica en cinco localizaciones: el pecho (esternón superior), la mitad del muslo en ambas piernas y ambos pies. La escala de los sensores es de $\pm 2 \mathrm{G}$. Además lleva una minicomputadora (59 $\mathrm{g}$ de peso) en la cintura donde a la que se conectan tres cables delgados y flexibles. De esta manera es capaz de diferenciar entre posturas sedentarias y movimientos activos.

La validación clínica de IDEEA ha sido publicada en 2010 por el New York Obesity Research Center (9), Universidad de Columbia sobre Investigación en obesidad. El IDEEA tiene las siguientes funciones principales:

- Graba y reproduce los cambios de movimiento y de postura del cuerpo sobre una base de $24 \mathrm{~h}$, almacenando decenas de millones de datos para futuros análisis. 
- Identifica más de 40 tipos de actividad física, incluyendo estar acostado, estar sentado, caminar, subir escaleras, correr y saltar.

\section{- Proporciona} grabaciones exactas de la aparición, duración y frecuencia de la actividad física $y$ otros acontecimientos importantes del día; llevando a cabo un análisis detallado de la actividad física y de los componentes de gasto de energía en categorías, distribuyéndolas en un período determinado de tiempo.

- Calcula la cantidad y la intensidad de la actividad física (por ejemplo, la velocidad de la marcha) utilizando técnicas tales como la inteligencia artificial $y$ redes neuronales, proporcionando los resultados más precisos jamás reportados para un dispositivo portátil.

- Proporciona resultados como la cantidad de trabajo mecánico y la estimación del gasto energético en un periodo determinado. Estos datos son fundamentales para el control del peso y la obesidad, los programas de capacitación fitness y/o salud y para centros de rehabilitación y evaluación funcional.

Dispositivos que combinan la acelerometría con otras mediciones fisiológicas

Muchos investigadores también han tratado de combinar la frecuencia cardíaca con acelerómetros (en monitores separados) mostrando mejoras significativas en la precisión de la predicción. Los detalles de los métodos que combinan la frecuencia cardíaca y la acelerometría han sido revisados por Strath et al. (10) y Brage et al. (11). La combinación de aceleración con la frecuencia cardíaca y otras mediciones en una sola unidad podría simplificar los procesos de aplicación, descarga de datos y sincronización. Con este nuevo enfoque, actualmente destacan dos dispositivos disponibles para los investigadores: el Actiheart y el SenserWear.

El Actiheart (Mini Mitter Co., Inc.) es un dispositivo que ha integrado un acelerómetro (uniaxial) con una señal de electrocardiografía, detectando simultáneamente la frecuencia cardiaca y el movimiento del cuerpo. Se ha demostrado que la Actiheart predice el gasto energético a partir de un modelo de acelerometría y frecuencia cardiaca significativamente más preciso que si se emplea cualquiera de los dos parámetros de manera aislada (11).

Por otro lado, el brazalete SenseWear (BodyMedia Inc., Pittsburgh, PA) es otro monitor disponible recientemente en el mercado y que está diseñado para que se lleve puesto en la parte superior del brazo. Los sensores internos de los que dispone incluyen un sensor de acelerometría, un sensor de flujo térmico, un sensor galvánico que registra la respuesta de la piel, un sensor de temperatura de la piel y un sensor de proximidad de la temperatura ambiente del cuerpo. El acelerómetro en el brazalete es de dos ejes que utiliza un dispositivo sensor 
microelectromecánico que mide el movimiento. El software del fabricante calcula el gasto de energía de un sujeto usando un algoritmo patentado que combina aceleración, flujo de calor y otros parámetros. Sin embargo, no está claro qué porcentaje de cada parámetro ( $>20$ parámetros totales de salida) contribuyen a la ecuación de predicción.

Intille et al. (12) han debatido recientemente la tendencia que hay que seguir para poder evolucionar en este sentido en los próximos 5 años:

- Dispositivos que sean capaces de registrar y almacenar en memoria interna el resumen por meses 0 datos en bruto $(+60 \mathrm{~Hz}$, tres ejes) durante 1 semana o más en una sola carga. Los dispositivos tendrán aproximadamente el mismo tamaño o más pequeño que los dispositivos existentes.

- Los investigadores deberían tener la capacidad de cambiar el enfoque de sus medidas, llegando a proporcionar datos que sean comunes a partir de las señales del acelerómetro. Los esfuerzos para definir las normas para la validación cruzada de cada dispositivo pueden agilizar estos cambios.

- Los sensores deberán ser suficientemente pequeños y fáciles de portar de modo que los participantes puedan llevar más de un sensor en diferentes partes del cuerpo o bajo la ropa de manera cómoda.

- Los sistemas pueden mejorar la detección de la cantidad y tipo de actividad usando algoritmos estadísticos de reconocimiento de patrones que utilizan, no sólo las características de movimiento de los acelerómetros, sino también la información de otros tipos de sensores (por ejemplo, la ubicación).

A su vez, a más largo plazo, se podría llegar a conseguir:

- Desarrollar un programa que pueda estar asociado a los teléfonos móviles, por medio de "app store", y así descargar los datos directamente.

- Emplear formas divertidas de enseñar a los participantes a utilizar y llevar los dispositivos mientras se realiza la recogida de datos.

- Detectar el nivel general de actividad física si se lleva en el bolsillo o en la cadera.

- Detectar los tipos específicos de actividad física como caminar, posturas e incluso actividades de resistencia si se utiliza con uno o más dispositivos inalámbricos adicionales que se comunican con el teléfono.

- Proporcionar a los participantes una retroalimentación audiovisual del sistema para saber si se está usando correctamente.

- Facilitar al participante un feedback con la información detectada de la actividad física con aplicaciones de vigilancia de la salud, gestión del tiempo o incluso juegos. 
- Transmitir los datos sobre la actividad física a los investigadores diariamente.

- Crear a largo plazo, para los investigadores, mapas de actividad superpuestos segundo a segundo con el lugar donde se realiza la actividad.

\section{- Ofrecer nuevas oportunidades} para crear intervenciones que influyan en el momento de toma de decisiones con la medida, con un feedback inmediato.

Intille et al. (12) informan que algunos de los dispositivos que están en uso actualmente, podrán estar con un funcionamiento idéntico durante los próximos 10 años. Los componentes electrónicos internos, firmware y protocolos inalámbricos de chips serán propensos a cambiar a medida que las antiguas tecnologías se vayan suplantando por la mejora de los dispositivos o por el descenso del coste económico. Para maximizar el efecto de la investigación en la actividad física que se lleva a cabo hoy en día, los investigadores deben esperar y planificar un cambio mientras explotan plenamente el potencia de los nuevos dispositivos.

\section{Referencias bibliográficas}

1. Armstrong N, Bray S. Primary school children is physical activity patterns during autumn and summer. Bulletin of Physical Education, 1990; 26: 23-26.

2. Rowlands $A V$, Eston RG, Ingledew DK. The relationship between activity levels, aerobic fitness, and body fat in 8- to 10yr-old children. Journal of Applied Physiology, 1999; 86:1428-1435.

3. Trost SG, Pate RR, Sallis JF, Freedson PS, Taylor WC, Dowda M, Sirad J. Age and gender differences in objectively measured physical activity in youth. Medicine and Science in Sports and Exercise, 2002; 334:350-355.

4. Martínez-Martínez J, Contreras OR, Aznar S, Lera A. Niveles de actividad física medido con acelerómetro en alumnos de $3^{\circ}$ ciclo de Educación Primaria: actividad física diaria y sesiones de Educación Física. Revista de Psicología del Deporte, 2012; 21 (1):117-123.

5. Butte NF, Ekelund U, Westerterp KR. Assessing physical activity using wearable monitors: measures of physical activity. Medicine and Science in Sports and Exercise, 2012; 44(1 Suppl 1):S5-S12.

6. Heil DP, Brage S, Rothney MP. Modeling physical activity outcomes from wearable monitors. Medicine and Science in Sports and Exercise, 2012; 44(1 Suppl 1):S50-60.

7. Strath SJ, Pfeiffer KA, Whitt-Glover MC. Accelerometer use with children, older adults, and adults with functional limitations. Medicine and Science in Sports and Exercise, 2012; 44(1 Suppl 1):S77-85.

8. Chen KY, Bassett DR, Jr. The technology of accelerometry-based activity monitors: current and future. Med Sci Sports Exerc, 2005; 37(11 Suppl):S490-500.

9. Kwon S, Jamal M, Zamba GK, Stumbo $P$, Samuel I. Validation of a novel physical activity assessment device in morbidly obese females. J Obes. 2010; 2010: pii: 856376

10. Strath SJ, Swartz AM, Bassett WL, O'Brien DR, King GA, Ainsworth BE. Evaluation of heart rate as a method for assessing moderate intensity physical activity. Med. Sci. Sports Exerc, 2000; 32(suppl):465-470.

11. Brage $S$, Brage N, Franks PW, Andersen LB, Froberg K. Branched equation modeling of simultaneous accelerometry and heart rate monitoring improves estimate of directly measured physical activity energy expenditure. J. Appl. Physiol., 1996; 96:343-351.

12. Intille SS, Lester J, Sallis JF, Duncan G. New Horizons in Sensor Development. Med. Sci. Sports Exerc., 2012; 44(1S):S24-S31. 